\title{
Estado nutricional, ingesta dietética y niveles de glicemia en estudiantes universitarios de Pedagogía en Educación Física \\ Nutritional status, dietary intake, and blood glucose levels in Physical Education students \\ Noemi Salvador Soler, José Gastón Bruneau Chávez, Andrés Esteban Roberto Godoy Cumillaf \\ Universidad Autónoma de Chile (Chile)
}

\begin{abstract}
Resumen. Los estudiantes universitarios se caracterizan por presentar hábitos alimentarios irregulares y poco saludables. En particular, los estudiantes de Pedagogía en Educación Física son sujetos más activos que otros universitarios, por lo que deberían presentar unos hábitos más saludables que la población general para obtener un óptimo rendimiento físico y académico. Sin embargo, estudios previos a esta investigación señalaron un alto porcentaje de estos estudiantes en hipoglicemia bajo condiciones de ayuno. El objetivo de investigación fue determinar el estado nutricional, la ingesta dietética y el nivel de glicemia de los estudiantes de esta carrera en Temuco (Chile). La muestra incluyó 56 estudiantes de la carrera de Pedagogía en Educación Física de primer ( $\mathrm{n}=30$ ) y tercer (n=26) año. Las variables estudiadas correspondieron al estado nutricional (índice de masa corporal, IMC), glicemia en ayuno e ingesta dietética (registro 24 horas). Los evaluados obtuvieron valores promedios de $23 \mathrm{~kg} / \mathrm{m}^{2}$ para el IMC. Se observaron diferencias estadísticamente significativas entre los cursos para la glicemia $(p<, 05)$. Destaca el elevado porcentaje de alumnos con un consumo de nutrientes por debajo de sus requerimientos, principalmente respecto a las calorías totales (41\%), a las grasas (61\%) y a los carbohidratos (25\%). Se concluye que la mayoría de los estudiantes evaluados se clasifican en un estado nutricional normal, presentan hipoglicemia y una estrategia alimentaria centrada en una ingesta alta de proteínas.
\end{abstract}

Palabras claves: educación física, estado nutricional, estudiantes, ingesta dietética, glicemia.

\begin{abstract}
University students usually present irregular and unhealthy eating patterns. Physical Education students are more active than other college students. Thus, they should display better healthy habits than the average population, mainly in order to achieve optimal physical and academic performance. However, studies previous to this investigation indicated that a high percentage of these students show hypoglycaemia under fasting conditions. The objective of this study was to determine the nutritional intake and blood glucose levels of university students from Physical Education. The sample included 56 students of Physical Education (Chile), who belonged to the first $(\mathrm{n}=30)$ and third $(\mathrm{n}=26)$ academic year. Body Mass Index (BMI), fasting blood glucose, and nutritional intake (24-hour dietary recall) were measured in each subject. The students showed an average BMI of $23 \mathrm{~kg} / \mathrm{m}^{2}$. Statistically significant differences were observed in the blood glucose levels $(p<.05)$ between students from different academic years. Outcomes highlighted a high percentage of students with dietary intake lower than the total calories (41\%), fats (61\%), and carbohydrates (25\%) required. On the contrary, 50\% of the students showed a protein intake higher than $120 \%$ of their nutritional requirements. In conclusion, most of the evaluated students were classified with a normal BMI, had hypoglycaemia, as well as a food strategy focused on high intake of proteins.
\end{abstract}

Key words: physical education, nutritional status, students, dietary intake, glycemia.

\section{Introducción}

Los estudiantes universitarios se caracterizan por presentar hábitos alimentarios irregulares y poco saludables, con una alta ingesta de alimentos ricos en lípidos y carbohidratos, un consumo excesivo de bebidas azucaradas y una baja ingesta en fibra dietética, con omisión de comidas y presencia de ayunos (Durán, Valdés, Godoy \& Herrera, 2015). Estos hábitos poco saludables han sido descritos tanto en el período escolar (Castillo, Tornero \& García, 2018) como durante el período universitario y estarían relacionados con la alta prevalencia de sobrepeso en Chile, particularmente entre los 17 a 24 años (MacMillan, 2007). Según ValdésBadilla, Godoy-Cumillaf, Herrera-Valenzuela \& Durán-Agüero (2015a), los estudiantes de Pedagogía en Educación Física no presentan patrones alimentarios distintos a otros estudiantes universitarios, pero en su mayoría sí cumplen con las recomendaciones de realización de actividad física en comparación con otros estudiantes universitarios (Pérez, Laíño, Zelarayan \& Márquez 2014). Es por ello que una ingesta dietética desbalanceada podría comprometer tanto su rendimiento físico como académico debido a que el glucógeno es el almacén de glucosa en el organismo y junto con la glicemia

Fecha recepción: 28-02-19. Fecha de aceptación: 03-06-19 Noemi Salvador Soler

noemi.salvador@uautonoma.cl sanguínea son los principales sustratos energéticos para el músculo y el sistema nervioso central (Peinado, Rojo-Tirado \& Benito, 2013; Iglesias, Planells \& Molina, 2019).

Según Sakata et al. (2001) privarse de tomar desayuno se ha asociado con un peor estado nutricional y un mayor riesgo de enfermedad cardiovascular. En ese sentido, los estudios previos de Valdés-Badilla, Salvador Soler, GodoyCumillaf, Carmona-López, Fernández \& Durán-Agüero (2015b) y Salvador, Godoy, Mella, Carmona \& Fernández (2016), resaltaron un elevado número de estudiantes de esta misma carrera universitaria en hipoglicemia bajo condiciones de ayuno. Ambos estudios sugieren que podría ser debido a un bajo consumo de hidratos de carbono, nutriente que sería esencial para ellos, considerando la actividad física que se les impone en sus asignaturas prácticas. Además, es necesario resaltar la alta frecuencia de ayuno que parece caracterizar a los estudiantes de Pedagogía en Educación Física según la investigación de Durán, Valdés, Godoy \& Herrera (2014) en la que se observó que solo un 30\% de estos estudiantes tomaban desayuno diariamente. Situación similar se reportó un año después, pero esta vez no solo con estudiantes de Pedagogía en Educación Física, sino que también de otras carreras universitarias (Valdés-Badilla et al., 2015a). Resultados similares han sido descritos por Sakamaki, Amamoto, Mochida, Shinfuku \& Toyama (2005), al observar que solo un 32,6\% de estudiantes universitarios de Corea tomaban desayuno. 
Teniendo en cuenta los antecedentes planteados, la presente investigación tiene como objetivo determinar el estado nutricional, la ingesta dietética y el nivel de glicemia de los estudiantes universitarios de Pedagogía en Educación Física de la Universidad Autónoma de Chile en Temuco.

\section{Material y método}

La investigación fue de enfoque cuantitativo, tipo no experimental y transversal y de diseño descriptivo. La muestra fue seleccionada bajo un criterio no probabilístico, que incluyó 56 estudiantes, 23 mujeres y 33 hombres, pertenecientes a primer y tercer año, cohortes $2014(\mathrm{n}=30)$ y 2012 $(n=26)$ respectivamente, de la carrera de Pedagogía en Educación Física de la Universidad Autónoma de Chile, sede Temuco. Como criterios de inclusión fueron considerados todos los estudiantes de primer y tercer año de la carrera que aceptaron participar en las evaluaciones y que firmaron el consentimiento informado que autoriza el uso de la información con fines científicos. Los criterios de exclusión fueron no asistir a alguna de las evaluaciones, no asistir en condición de ayuno para la medición de glicemia, consumir suplementos alimentarios o presentar alguna enfermedad que pueda influir en los resultados de los test, como resistencia a la insulina o diabetes. El estudio fue aprobado por el Comité de Ética de la Universidad Autónoma de Chile ( $\left.\mathrm{N}^{\circ} 008\right)$ y desarrollado siguiendo lo expuesto en la Declaración de Helsinki.

La valoración de la glicemia se realizó a cada estudiante después de 12 horas de ayuno mediante un glucómetro Accutrend ${ }^{\circledR}$ Plus (Roche, Chile), instrumento que otorga la lectura de la glicemia a partir de una muestra de sangre capilar aplicada en una tira reactiva. Se clasificaban en hipoglicemia cuando las concentraciones de glucosa en sangre estaban por debajo de $70 \mathrm{mg} / \mathrm{dl}$ (American Diabetes Association, 2005). También fue registrada la edad y evaluadas las variables antropométricas de peso corporal, por medio de una balanza digital (Scale-tronik, USA) con precisión de 0,1kg, y la altura, con estadiómetro (Seca 220, Alemania), con precisión de $1 \mathrm{~mm}$. Todo según el protocolo expuesto por la sociedad internacional para el avance de la kineantropometría (ISAK). Posteriormente se calculó el índice de masa corporal (IMC) para categorizar el estado nutricional de acuerdo a los criterios establecidos por la Organización Mundial de la Salud (OMS) en el año 2017. Todas las medidas fueron tomadas por dos evaluadores certificados nivel III por la ISAK.

Para realizar la valoración de la ingesta dietética se utilizó un registro nutricional de 24 horas (Recordatorio de 24h), instrumento que permite valorar el promedio de dicha ingesta (Block, 1982). Días antes de la evaluación, los estudiantes recibieron instrucción por parte de los investigadores sobre las medidas de los alimentos ingeridos en forma de proporciones caseras y de las características del cuestionario para garantizar su comprensión. Posteriormente, el cuestionario fue aplicado en días alternos y correspondientes a dos días de clases (martes y jueves) por estudiante, revisando individualmente los casos de dudas, ambigüedad u omisión de datos. Se obtuvieron los valores promedio de macronutrientes y calorías totales mediante el programa de cálculo nutricional denominado ENCUESTA.EXE que emplea la base de datos nutricional utilizada por la Universidad de Chile y el Instituto de Nutrición y Tecnología de los Alimentos (INTA). Además, el programa clasifica los resultados en función de los requerimientos diarios para cada individuo al considerar su talla, peso, sexo y nivel de actividad física, quedando por tanto en las siguientes categorías $<80 \%$, 80-120\% y $>120 \%$.

Todas las valoraciones de los estudiantes fueron realizadas en las dependencias de la Universidad Autónoma de Chile, sede Temuco.

\section{Análisis estadístico}

Los estadísticos descriptivos (media y desviación estándar) y la inferencia estadística fue realizada con el software Statistical Package for Social Science (SPSS) versión 22.0. La distribución normal de las variables edad, peso, talla, IMC y glicemia se analizó mediante la prueba de ShapiroWilks. Las diferencias tanto entre generaciones (2012 y 2014) como entre sexo para cada una de las variables fue evaluada con el test t para muestras independientes (nivel de significancia de $p<, 05$ ) en aquellas variables que cumplieron con el criterio de normalidad, en caso contrario se utilizó el test de U Mann-Whitney.

\section{Resultados}

En la Tabla 1 se puede observar que los estudiantes evaluados tienen un promedio para la edad de 21 años, un peso de $67 \mathrm{~kg}$, una talla de 1,69 mt, un IMC de $23 \mathrm{~kg} / \mathrm{m}^{2}$ y una glicemia en ayunas de $67 \mathrm{mg} / \mathrm{dl}$ para la cohorte 2012, mientras que para la 2014 los valores promedios fueron para la edad 19 años, un peso de 65,6 kg, una talla de 1,69 mt, un

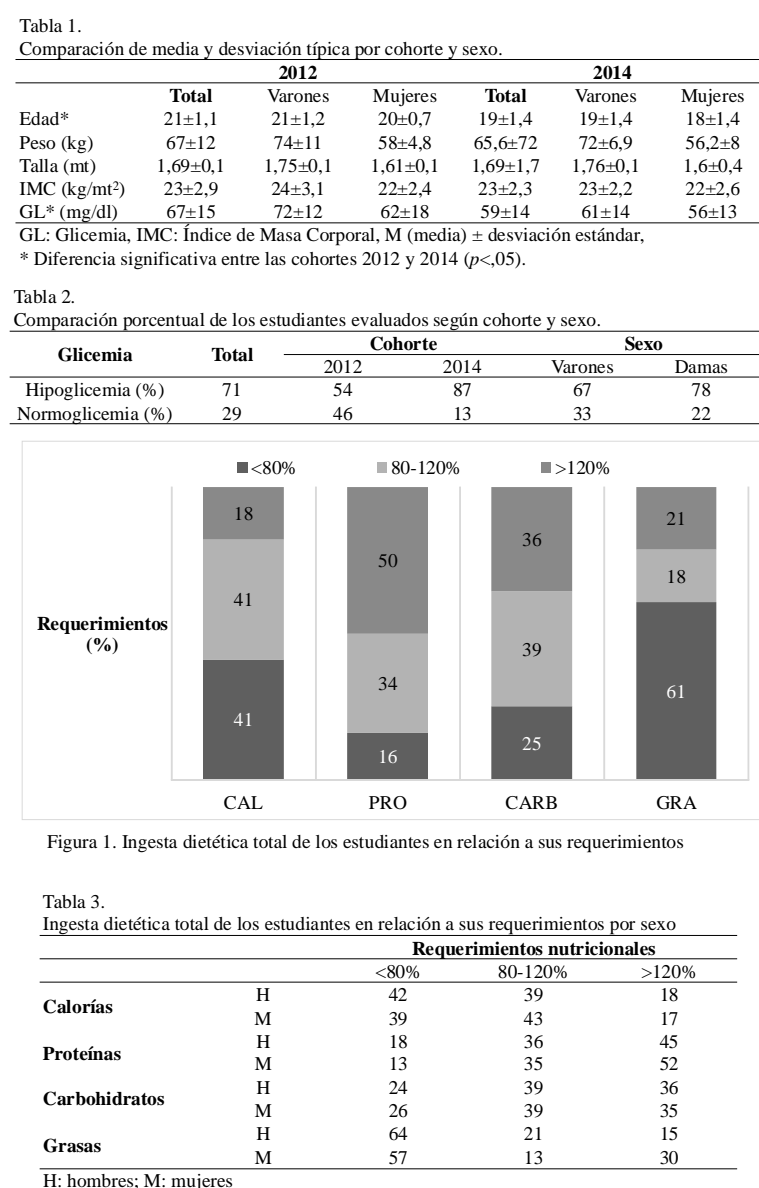


IMC de $23 \mathrm{~kg} / \mathrm{mt}^{2}$ y una glicemia en ayuno de $59 \mathrm{mg} / \mathrm{dl}$. La única diferencia estadísticamente significativa observada entre cohortes fue para las variables edad y glucosa, siendo la cohorte del año 2014, la que mostró menores niveles de glucosa en sangre.

Respecto al total de evaluados, un 71\% de los estudiantes se encontraron en situación de hipoglicemia y solo un $29 \%$ en normoglicemia, tal como se puede observar en la Tabla 2. Al comparar por cohorte los porcentajes de hipoglicemia, se observa que ésta es mayor en los alumnos de primer año (87\%, cohorte 2014) y que la prevalencia es mayor en damas que en varones (Tabla 2).

Con relación a la evaluación de la ingesta dietética de los estudiantes (Figura 1), se observó una elevada prevalencia (61\%) con una ingesta de grasa dietética por debajo del 80\% de sus requerimientos diarios, seguida por los carbohidratos (25\%) y en último término por las proteínas (16\%). Por el contrario, un $50 \%$ de los estudiantes mostraron ingestas superiores a sus requerimientos (>120\%) en proteínas y un $36 \%$ para los carbohidratos.

Al comparar entre sexos no se observaron diferencias estadísticamente significativas entre las ingestas de hombres y mujeres, tanto para las calorías totales como para los tres macronutrientes, resaltando nuevamente la alta prevalencia en ambos sexos de una ingesta por debajo del 80\% de sus requerimientos diarios en grasa dietética (Tabla 3).

\section{Discusión}

La presente investigación tiene como principal hallazgo, que gran parte de los evaluados presentan hipoglicemia. Además, el $60 \%$ de los evaluados tienen una ingesta por debajo del $80 \%$ de sus requerimientos diarios de grasa y el $50 \%$ un consumo mayor al $120 \%$ de los requerimientos de proteínas.

Llama la atención el gran número de estudiantes con hipoglicemia, lo que puede estar relacionado con el efecto fisiológico que provoca la actividad física sobre el componente evaluado (López \& Fernández, 2006) y/o a que no consumen los nutrientes necesarios para cubrir sus demandas energéticas (Durán et al., 2015). En ese sentido, las diferencias significativas en el nivel de glicemia de los estudiantes entre las generaciones 2012 y 2014, tercer y primer año de carrera respectivamente, podrían estar relacionadas con el mayor número de actividades de carácter práctico en el Plan de Estudios de primer año, o a que estos estudiantes aún no cuentan con los conocimientos suficientes sobre qué nutrientes consumir de acuerdo con el tipo de actividad que realizan, situación que los de tercer año ya manejan debido a los conocimientos que han adquirido.

Por otra parte, la prevalencia de hipoglicemia fue mayor en damas que en varones, situación que ha sido descrita en otros estudios (Durán et al., 2015; Valdés-Badilla et al., 2015b; Salvador et al., 2016) y tiene relación con el metabolismo de las damas, ya que poseen una mayor sensibilidad a la insulina en todo el cuerpo en comparación con los hombres, quienes tienden a oxidar más lípidos (Magkos, Wang \& Mittendorfer, 2010; Varlamov, Bethea \& Roberts, 2015). Es importante considerar que independientemente de quienes tengan mayor prevalencia o porque se produzca la hipoglicemia, esta situa- ción debe ser prevenida y corregida, ya que su déficit podría traer consecuencias negativas severas, debido a que está demostrado que la hipoglicemia se relaciona con cambios en la estructura cerebral y la función de la memoria (Kirchhoff et al., 2013).

Para el estado nutricional, los hombres tienen valores más altos que las mujeres, situación similar a lo reportado en otras investigaciones (Iglesias, Mata, Pérez, Hernández, García-Chico \& Papadaki, 2013; Durán-Aguero et al., 2015), a pesar de esto, la media de los estudiantes se encuentra dentro de la clasificación de normal (OMS, 2017), lo cual es positivo en comparación con lo señalado por el Ministerio del Deporte de Chile (2016) que reporta un 67\% de la población chilena mayor de 15 años con sobrepeso y obesidad. Y porque altos valores de IMC se señalan como el mejor predictor de muerte por factores cardiovasculares, sobre otros índices (Ortega, Sui, Lavie \& Blair, 2016).

Respecto a las calorías totales consumidas, un 41,1\% está por debajo de lo recomendado, similar a lo reportado en otros estudiantes universitarios (Iglesias et al., 2013). Sin embargo, estos resultados en el caso de los estudiantes de Educación Física deberían considerarse de forma especial, ya que la actividad metabólica generada durante la actividad física y el entrenamiento puede suponer un problema en la homeostasis cuando las necesidades energéticas no están cubiertas (Peinado et al., 2013). Además, según Pinheiro, Canaan \& Gonçalves (2008) la privacion energetica puede llevar a una disminucion de las reservas de glucogeno hepatico, hipoglicemia y aumento del apetito.

En lo referente al consumo de proteínas, se encontró que la mitad de los evaluados tiene un consumo por encima de sus requerimientos, lo cual es similar a lo reportado en otras investigaciones que trabajaron con estudiantes universitarios (Oliveras, Nieto, Agudo, Martínez, López \& López, 2006; Vargas-Zárate, Becerra-Bulla \& Prieto-Suarez, 2010). De acuerdo con lo reportado por Bastardo, Angarita, Quintero, Rojas, Rodríguez \& Márquez (2008), en la mayoría de los países industrializados y en vías de desarrollo, el consumo de proteínas excede las recomendaciones establecidas internacionalmente, lo que de acuerdo con la OMS (2017)se debe principalmente a la ingesta de dietas basadas en la carne.

Durante mucho tiempo ha existido la creencia de que una ingesta excesiva de este nutriente repercute en alteraciones del balance ácido base y electrolítico, del metabolismo óseo, de la función renal y de la función endocrina (López \& Fernández, 2006; López-Luzardo, 2009). Por este motivo se recomendaba que el consumo de proteínas no representase más del 15-20\% del consumo calórico diario total. Sin embargo, los análisis basados en la evidencia de Phillips, Chevalier \& Leidy (2018) mostraron que estas creencias no presentan fundamento e incluso sugieren una ingesta de proteínas mayor a lo recomendado hasta ahora, pasando de $0,8 \mathrm{~g} / \mathrm{kg}$ día (Institute of Medicine 2005) a 1,2-1,6 g/kg día con el objetivo de promover el envejecimiento saludable, el control de peso y el rendimiento atlético. Además, según Paddon-Jones \& Rasmussen (2009) si bien no existe problema, en términos de salud, en que la ingesta sea superior a lo recomendado actualmente, un exceso de aminoácidos en la dieta más allá de lo que el músculo necesita para estimular al máximo la 
síntesis de proteínas no se relaciona con su inmediata incorporación al tejido muscular y por lo tanto puede ser considerado como un nutriente desperdiciado. Es decir, cuando se supera el umbral de ingesta la proteína pierde su efecto anabólico. Según López \& Fernández si el individuo tiene como objetivo aumentar la masa muscular, debería optar por una alimentación equilibrada que permita aumentar el aporte calórico total y no el procedente únicamente de las proteínas. De acuerdo con Carbone, McClung \& Pasiakos (2012) un balance energético negativo a largo término disminuye la masa corporal debido a la pérdida no solo de masa grasa sino también del músculo esquelético. Esta disminución de la masa muscular sería causada por un desequilibrio entre la síntesis y la degradación de proteínas musculares, que además estaría asociada a una gran cantidad de consecuencias negativas tales como la disminución en el recambio de proteínas, un menor rendimiento físico y un mayor riesgo de lesiones (Carbone, McClung \& Pasiakos 2012). Estos mismos autores en su investigación sugirieron que la ingesta de proteínas por encima de lo requerido podría ser un factor protector para la conservación de la masa muscular durante el déficit de energía. Sin embargo, recientemente dichos autores indicaron que a medida que aumenta la magnitud del déficit de energía, la eficacia de las dietas altas en proteínas para mitigar las pérdidas de masa libre de grasa disminuye (Carbone, McClung \& Pasiakos, 2018). Por tanto, los estudiantes de Pedagogía en Educación Física no deberían solo preocuparse de consumir proteínas sino más bien tratar de aumentar las calorías totales en base al consumo de grasas saludables.

Respecto al consumo de carbohidratos y grasas, principales sustratos energéticos en la actividad física, un 39\% de los estudiantes consume una cantidad de carbohidratos de acuerdo con sus necesidades, lo cual es similar a lo encontrado en otros sujetos universitarios evaluados (VargasZárate et al., 2010). Sin embargo, es importante destacar que un 25\% no alcanzó a cubrir el $80 \%$ de sus requerimientos lo cual podría dificultar la repleción de las reservas de glucógeno. Para el consumo de grasas, un $60 \%$ de los estudiantes presenta una ingesta que no alcanza al $80 \%$ de sus requerimientos, lo cual podría ser debido a una mayor exigencia calórica en los estudiantes de la carrera de educación física, ya que además muchos de ellos realizan entrenamientos deportivos. Por tanto, las exigencias serían distintas a cualquier otro tipo de estudiante universitario siendo así esperable que los resultados observados en otras investigaciones sean distintos, como en el trabajo de Oliveras et al. 2006, en las que se determinó un consumo alto de grasas. Además, los resultados aquí reportados sugieren que los estudiantes evaluados estarían dando más importancia al consumo de carbohidratos y proteínas sobre el de la grasa dietética, posiblemente debido a que en nutrición deportiva se enfatiza el consumo de estos dos macronutrientes, el primero como combustible energético y el segundo por su rol en la reparación y generación de masa corporal (Helms, Aragón \& Fitschen, 2014; Marins et al., 2018). Sería por tanto necesario recomendar a los estudiantes evaluados que aumentasen el consumo de grasas saludables no solo para mantener sus niveles de glicemia sino porque además son un componente necesario para una dieta saludable, debido a que proporcionan energía, son imprescindibles para la formación de nue- vas membranas celulares y para la asimilación de algunas vitaminas (Dietitian of Canada, 2006). Por tanto, el elevado porcentaje de alumnos en hipoglicemia podría ser explicado debido a que la mayoría de los estudiantes evaluados en esta investigación poseen hábitos alimentarios inadecuados observándose principalmente un bajo consumo de grasas, que se ve reflejado también en el porcentaje bajo de calorías totales consumidas, y centrando su alimentación en el consumo de proteínas.

Las fortalezas del estudio radican en la utilización de un cuestionario (registro 24 horas) e instrumento (sangre capilar) confiables y válidos, además de describir una realidad que afecta a la mayoría de los estudiantes de la Carrera de Educación Física de la Universidad Autónoma de Chile en la sede Temuco y que es necesario poner de manifiesto para evitar posibles problemas de rendimiento académico y salud. Tal y como sugieren Poblete-Valderrama, Linzmayer, Matus, Garrido y Flores (2018) la labor del docente en los procesos de formación inicial de profesores debe considerar el rol de la educación física en relación con la formación integral del alumno.

Nuestro estudio también tiene algunas limitaciones que deben ser mencionadas, debido a que el marco muestral de estudiantes de esta carrera en Temuco es pequeño, la elección de la muestra fue no probabilística, lo cual no permitió realizar inferencias de causalidad para explorar que factores dietéticos influyen en la glicemia.

Se recomienda que los estudiantes puedan contar con el apoyo de toda la comunidad educativa implícita en el proceso de formación de estos futuros profesionales, ya que no basta solo con generar programas pedagógicos que los orienten a mejorar sus hábitos alimentarios con el objetivo de evitar la hipoglicemia y sus consecuencias, sino que es necesaria una reestructuración del Plan de Estudios, para que no se concentre la mayor carga de asignaturas prácticas en el primer año.

\section{Conclusiones}

Se concluye que la mayoría de los estudiantes de Pedagogía en Educación Física evaluados se clasifican en un estado nutricional normal, presentan hipoglicemia y una estrategia alimentaria centrada en una ingesta alta de proteínas y baja en grasas, repercutiendo esto último en un detrimento del aporte calórico total. La prevalencia de hipoglicemia fue mayor en los estudiantes de primer año que en los de tercero, por lo que consideramos necesaria una futura investigación que incluya la aplicación de una intervención educativa, con evaluación pre- y post- en el primer año de la carrera, para determinar el nivel de conocimiento de los estudiantes acerca de las consecuencias del ayuno, la hipoglicemia y de un balance energético negativo.

\section{Agradecimientos}

A los estudiantes de la carrera de Pedagogía en Educación Física de la Universidad Autónoma de Chile que participaron en las evaluaciones.

\section{Referencias}

American Diabetes Association. (2005). Defining and reporting 
hypoglycemia in diabetes: a report from the American Diabetes Association Workgroup on Hypoglycemia. Diabetes Care, 28(5), 1245-9. doi:10.2337/diacare.28.5.1245

Bastardo, G., Angarita, C., Quintero, Y., Rojas, L., Rodríguez, L.C. \& Márquez, J.L. (2008).

Block, G. (1982). A review of validations of dietary assessment methods. American Journal of Epidemiology, 115(4), 492-505.

Carbone, J. W., McClung, J. P. \& Pasiakos, S. M. (2012). Skeletal Muscle Responses to Negative Energy Balance: Effects of Dietary Protein. Advances in Nutrition, 3(2), 119-126. doi:10.3945/ an.111.001792

Carbone, J. W., McClung, J. P. \& Pasiakos, S. M. (2018). Recent Advances in the Characterization of Skeletal Muscle and Whole-Body Protein Responses to Dietary Protein and Exercise during Negative Energy Balance. Advances in Nutrition, nmy087. doi:10.1093/advances/ nmy087

Castillo Viera, E., Tornero Quiñones, I., \& García Araujo, J. (2017). Relación entre actividad física, alimentación y familia en edad escolar (Relationship between physical activity, nutrition, and family in school-aged youth). Retos, 34, 85-88. Recuperado de https:// recyt.fecyt.es/index.php/retos/article/view/52782

Consumo de hierro y otros nutrientes en la dieta de preescolares residentes en Apartaderos, Escagüey y Pueblo Llano (Mérida, Venezuela). Revista Facultad de Medicina ULA, 17, 41-6.

Durán, A. S., Valdés B. P., Godoy, C. A. \& Herrera, V. T. (2014). Hábitos alimentarios y condición física en estudiantes de pedagogía en educación física. Revista chilena de nutrición, 41(3), 251-259. doi:10.4067/ S0717-75182014000300004

Durán, S., Valdés B. P, Godoy, C. A. \& Herrera, V. T. (2015). Consumption of fruits and its association with nutritional status in chilean university students career of physical education. Nutrición Hospitalaria, 31(5), 2247-52. doi: 10.3305/nh.2015.31.5.8617.

Helms, E., Aragón, A. \& Fitschen, P. (2014). Evidence-based recommendations for natural bodybuilding contest preparation: nutrition and supplementation. Journal of the Internacional Society of Sports Nutrition, 11, 1-20. doi: 10.1186/1550-2783-11-20.

Iglesias, A., Planells, E., \& Molina López, J. (2019). Prevalencia de sobrepeso y obesidad, hábitos alimentarios y actividad física y su relación sobre el rendimiento académico. Retos, 36, 167-173. Recuperado de https://recyt.fecyt.es/index.php/retos/article/view/66873.

Iglesias, M., Mata, G., Pérez, A., Hernández, S., García-Chico, R. \& Papadaki, C. (2013). Estudio nutricional en un grupo de estudiantes universitarios madrileños. Nutrición Cliìnica y Dietética Hospitalaria, 33(1), 23-30.

Institute of Medicine. 2005. Dietary Reference Intakes for Energy, Carbohydrates, Fiber, Fat, Fatty Acids, Cholesterol, Protein and Amino Acids. The National Academies Press, Washington, DC, USA.

Kirchhoff, B., Lugar, H., Smith, S., Meyer, E., Perantie, D., Kolody, B., ... \& Hershey, T. (2013). Hypoglycaemia-induced changes in regional brain volume and memory function. Diabetic Medicine, 30(4), e151-e156. doi: 10.1111/dme.12135.

López, C \& Fernández, V. (2006). Fisiología del ejercicio. Madrid, España: Panamericana.

López-Luzardo, M. (2009). Las dietas hiperproteícas y sus consecuencias metabólicas. Anales Venezolanos de Nutrición, 22(2), 95-104.

MacMillan, N. (2007). Valoración de hábitos de alimentación, actividad física y condición nutricional en estudiantes de la pontificia universidad católica de Valparaíso. Revista Chilena de Nutrición, 34(4), 3306. doi: 10.4067/S0717-75182007000400006

Magkos, F., Wang, X. \& Mittendorfen, B. (2010). Metabolic actions of insulin in men and women. Nutrition, 26(7-8), 686-693. doi: 10.1016/ j.nut.2009.10.013

Marins, J., Pereira, L., Amorin, P., Arnaiz-Lastras, J., Sillero-Quintana, M. \& Alfenas, C. (2018). Suplementos de carbohidratos durante un ejercicio: Efectos sobre los electrolitos y glucosa. Revista Internacional de Medicina y Ciencias de la Actividad Física y el Deporte, 18, 269-287. doi: 10.15366/rimcafd2018.70.005

Ministerio del Deporte. (2016). Encuesta nacional de actividad física y deportes en la población chilena de 18 años y más. Recuperado de http://www.mindep.cl/wp-content/uploads/2016/07/INFORME-FINAL-ENCUESTA-DEPORTES-COMPLETO_.pdf

Oliveras, M., Nieto, P., Agudo, E., Martínez, F., López, H. \& López, M. (2006). Evaluación nutricional de una población unive stranta. Nutr

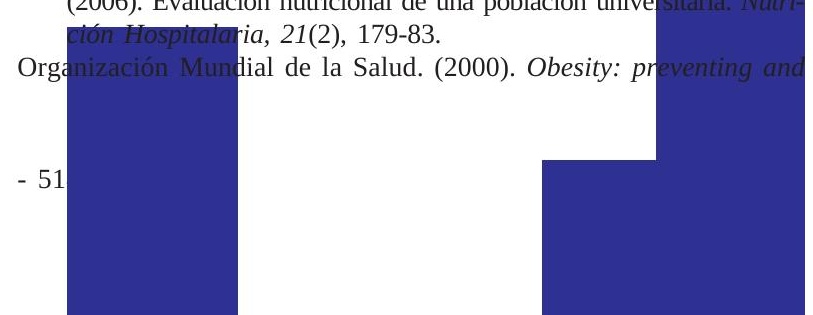

managing the global epidemic. Report of a WHO Consultation. Geneva: World Health Organization. Recuperado de https:// www.who.int/nutrition/publications/obesity/WHO_TRS_894/en/

Ortega, F., Sui, X., Lavie, C. \& Blair, S. (2016). Body Mass Index, the Most Widely Used But Also Widely Criticized Index: Would a Criterion Standard Measure of Total Body Fat Be a Better Predictor of Cardiovascular Disease Mortality?. Mayo Clinic Proceedings, 91(4), 443-455. doi: 10.1016/j.mayocp.2016.01.008.

Paddon-Jones, D. \& Rasmussen, B. B. (2009). Dietary protein recommendations and the prevention of sarcopenia. Current opinion in clinical nutrition and metabolic care, 12(1), 86-90. doi: 10.1097/ MCO.0b013e32831cef8b.

Peinado, A.B., Rojo-Tirado, M.A. \& Benito, P.J. (2013). El azúcar y el ejercicio físico: su importancia en los deportistas. Nutrición Hospitalaria, 28(Supl 4), 48-56.

Pérez Ugidos, G., Lanío, F. A., Zelarayán, J. \& Márquez, S. (2014). Actividad física y hábitos de salud en estudiantes universitarios argentinos. Nutrición Hospitalaria, 30(4), 896-904. doi: 10.3305/ nh.2014.30.4.7641.

Phillips, S., Chevalier, S. \& Leidy, H. (2016). Protein «requirements» beyond the RDA: implications for optimizing health. Applied Physiology, Nutrition and Metabolism, 41, 565-572. doi: 10.1139/ apnm-2015-0550.

Pinheiro, A., Canaan, F. \& Gonçalves, R. (2008). Insulemia, ingesta alimentaria y metabolismo energético. Revista chilena de nutrición, 35(1), 18-2. doi:10.4067/S0717-75182008000100003

Poblete-Valderrama, F., Linzmayer, L., Matus, C., Garrido, A. \& Flores, C. (2018) Percepción de estudiantes de pedagogía en educación Física hacia sus profesores. Retos, 33, 143-147. Recuperado de https:// recyt.fecyt.es/index.php/retos/article/view/51934/35804

Sakamaki R., Amamoto R., Mochida Y., Shinfuku N. \& Toyama K. (2005). A comparative study of food habits and body shape perception of university students in Japan and Korea Nutrition Journal, 4, 3140. doi: 10.1186/1475-2891-4-31

Sakata K., Matumara Y., Yoshimura N., Tamaki J., Hashimoto T., Oguri S., Okayama A. \& Yanagawa H. (2001). Relationship between skipping breakfast and cardiovascular disease risk factors in the national nutrition survey data. Nipon Koshu Eisei Zasshi, 48, 837-41.

Salvador, N., Godoy, A., Mella, J., Carmona, M. \& Fernández, J. (2016). Estado nutricional, riesgo cardiovascular y nivel de glicemia de estudiantes universitarios de pedagogía en educación física. Nutrición Hospitalaria, 33(2), 289-291. doi: 10.20960/nh.105

Thomas, D., Erdman, K. \& Burke, L. (2016). Position of the Academy of Nutrition and Dietetics, Dietitians of Canada, and the American College of Sports Medicine: Nutrition and Athletic Performance. Journal of the Academy of Nutrition and Dietetics, 116(3), 501-528. doi: 10.1016/j.jand.2015.12.006.

Valdes-Badilla, P., Godoy-Cumillaf, A., Herrera-Valenzuela, T. \& DuránAgüero, S. (2015a). Comparación en hábitos alimentarios y condición física entre estudiantes de educación física y otras carreras universitarias. Nutrición Hospitalaria, 32(2), 829-836. doi: 10.3305/ nh.2015.32.2.9194

Valdés-Badilla, P., Salvador Soler, N., Godoy-Cumillaf, A., Carmona-López, M. I., Fernández, J.J. \& Durán-Agüero, S. (2015b). Somatotipo, estado nutricional y nivel de glicemia de estudiantes de educación física. Nutrición Hospitalaria, 32(3), 1261-6. doi: /10.3305/ nh.2015.32.3.9402

Vargas-Zárate, M., Becerra-Bulla, F. \& Prieto-Suarez, E. (2010). Evaluación de la ingesta dietética en estudiantes universitarios. Bogotá, Colombia. Revista de Salud Pública, 12(1), 116-125. doi: 10.1590/ S0124-00642010000100011

Varlamov, O., Bethea, C. \& Roberts, C. (2015). Sex specific differences in lipid and glucose metabolism. Frontier in Endocrinology (Lausanne), 19(5), 1-7. doi: 10.3389/fendo.2014.00241.

Noemi Salvador Soler1, José Gastón Bruneau Chávez2 y Andrés Godoy Cumillaf2

1 Instituto de Ciencias Biomédicas, Facultad de Ciencias de la Salud, Universidad Autónoma de Chile, Temuco, Chile.

2 Facultad de Educación, Universidad Autónoma de Chile, Temuco,

Chile. 